\title{
Modeling and simulation of a multi-functional high- lift actuation system based on key performance data
}

\author{
Andreas Schäfer ${ }^{1 *}$, René Hollmann ${ }^{2}$, Oliver Bertram ${ }^{1}$ \\ ${ }^{1}$ German Aerospace Center (DLR), Institute of Flight Systems, Lilienthalplatz 7, 38108 Braunschweig, Germany; \\ *a.schaefer@dlr.de \\ ${ }^{2}$ German Aerospace Center (DLR), Institute of Flight Systems, Cornelius-Edzard-Straße 15, 28199 Bremen, Germany
}

\begin{abstract}
The rising complexity of flight control systems leads to a huge amount of additional testing. A major quantity of malfunctions is identified during system integration activities at a late stage in the development process. In order to reach high maturity as early as possible especially on the overall system level, virtual testing methods become increasingly important. This paper describes the development of a flight control actuation systems library based on key performance data. The library is implemented in the modeling language Modelica and enables the simulation and analysis of state-of-the-art high-lift actuation systems including the main mechanical failure cases. In addition, a script-based preprocessing is implemented that minimizes the parameterization effort for different test cases.
\end{abstract}

\section{Introduction}

The development of safety-critical flight control systems requires a high number of physical tests during the entire design process $[1,2,3]$. Since the system complexity increases due to the implementation of new functions and the development of multi-functional movables, the number of system requirements rises. As a result, the test activities need to be further expanded [2]. In the beginning of the system design process, small component and equipment test benches perform a decisive role. These test benches are required to verify the performance as well as to evaluate aspects such as endurance and fatigue. This verification is usually provided by the suppliers of the components and equipment [4]. The tests are also used to identify the main characteristics such as moments of inertia and frictional losses of the equipment. These identified parameter values are part of the required deliverables and are provided to the original equipment manufacturer (OEM) as so-called key performance data.

An essential verification effort is performed by the OEM on so-called zero-means. Zero-means are test rigs that represent an entire aircraft system. Up to 90 percent of all faults are detected on the system level using such test means [3]. However, zero-means are only available late in the development process so that found faults may lead to significant modification costs and delays. The dilemma is enhanced by a growing number of interactions, even beyond system boundaries, due to more complex systems with an increased number of functions. In order to overcome this challenge as well as to minimize the physical testing effort in general, currently an important research focus lies on virtual testing methods [5,6,7]. Reliable models and accurate parameter values are essential prerequisites for virtual testing. The required knowledge can be gained by design and test activities performed on the component or equipment level. This way, valid data can be used to evaluate system level requirements much earlier in the development process than with zero-means. In order to demonstrate such an approach, the modeling of equipment of a highlift actuation system based on key performance data is presented in this work.

Using key performance data, nonlinear models with lumped parameters are implemented. The equationbased, object-oriented, multi-domain modeling language Modelica [8] is chosen for this purpose. The utilization of Modelica ensures high flexibility in modeling and enables the realization of a high degree of automatization for model generation and parameterization. In contrast to [9], the modeling based on actual key performance data enables, for example, the usage of models independent of the system supplier and minimizes the effort of mapping parameter sets to simulation models, mitigating modeling errors. In order to keep the modeling as well as the verification and validation effort as low as possible, the models of different equipment are based on common component models. A component model specifies a physical characteristic such as frictional losses, backlash or torsional stiffness. Since the 
required model structure of an equipment model is equal for similar equipment types embedded in different aircraft, the same equipment model can be used in different aircraft types. The possibility to reuse validated models is a crucial prerequisite to keep the modeling effort low and to exploit the full potential of virtual testing. The key performance data provide a good basis to achieve this goal. In addition to the nominal characteristics, the mechanical faults disconnection and jamming are implemented in the flight control actuation systems library. Finally, an approach for test case parameterization is introduced and some simulation results are discussed.

\section{Flight Control Actuation Systems Library}

The model library developed in the context of this work allows the modeling and simulation of a state-of-the-art multi-functional high-lift actuation system. Such a system is illustrated in Figure 1. The main difference to a classical high-lift system is the additional drive unit installed in the transmission between the inner and outer flap. The electrically-powered active differential gearbox (ADGB) enables a fully independent motion of the flaps [10]. The differential flap setting is applied to optimize the load distribution of the wing. As a result, the weight of the wing structure can be reduced [11].

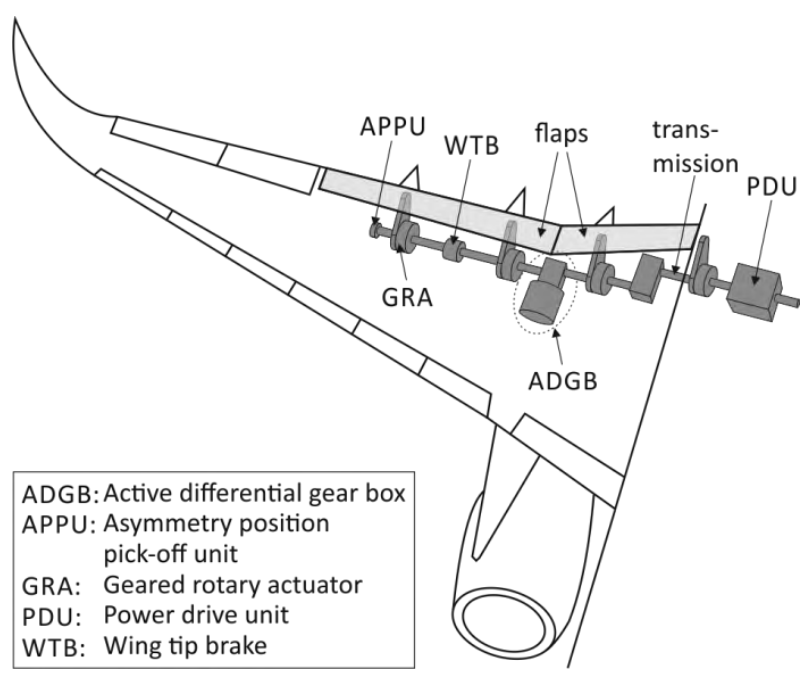

Figure 1: Architecture of a state-of-the-art multi-functional high-lift system [12]

The flight control actuation systems library is implemented in the equation-based and object-oriented modeling language Modelica. A major difference to a block- oriented approach is that it is not required to specify a certain data-flow direction. Such an acausal feature reduces the modeling effort of physical systems and enables the most possible flexibility and reusability [8]. These and further differences between the two modeling concepts are pointed out in [8] using a simple electric circuit and in [13] modeling a mechanical system. Nevertheless, block-oriented modeling is also supported by the Modelica language. A component-based approach is applied in the implementation of the model library. The required equipment models are created by connecting the developed component models or component models from the Modelica Standard Library. Consequently, the flight control library consists of two main packages: the component package and the equipment package. An overview of a selection of relevant component and equipment models of a high-lift actuation system is presented in the following subsections.

\subsection{Component Models}

A state-of-the-art high-lift actuation system consists of mechanical, hydraulic and electric equipment. Nevertheless, most of the equipment is from the mechanical domain due to the centralized power generation. The central drive unit is mechanically connected to all trailing edge devices via shafts, gearboxes and joints. The key performance data for mechanical equipment can include the following parameters:

- Moment of inertia

- Torsional stiffness

- Mechanical backlash

- Frictional losses

The mechanical equipment is characterized at least by its moment of inertia and torsional stiffness. The torsional stiffness is modeled by a spring-damper system. Since the damping constant is not part of the key performance data yet, an estimated value is assumed based on empirical data for all equipment models. For more accurate simulation results the damping constant should also be part of the key performance data in the future. In addition, mechanical backlash is considered for the most mechanical equipment models. All parameter values are identified with respect to the input (drive) side of the equipment. The required component models are available in the Modelica Standard Library (Inertia, SpringDamper and ElastoBacklash) [14].

Frictional losses are another important characteristic of mechanical equipment of a high-lift system. The 
speed-dependent frictional losses are characterized by a breakout torque and a running drag torque. The breakout torque is also referred to as break-away torque and must be overcome to initiate motion [15]. The running drag torque is defined by a drag torque value at referenced low system speed and a drag torque value at nominal system speed. Between those two points a linear increase of the drag torque is assumed approximately. It is presumed that the referenced low system speed is very close to zero. After reaching the nominal system speed, the running drag torque is kept constant. The model of the speed dependent friction torque is illustrated in Figure 2.

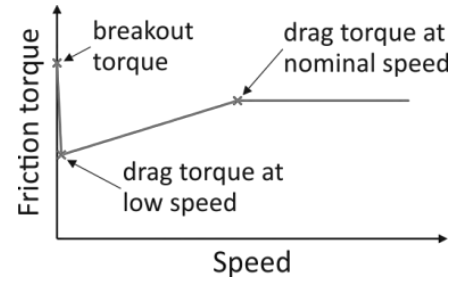

Figure 2: Model of the speed dependent friction torque

In addition to the speed-dependent losses, a loaddependent friction torque characterized by an efficiency value represents, for instance, the meshing friction of a gearbox. If required, an efficiency value for opposing loads and one for aiding loads can be defined. In context of high-lift actuation systems, this distinction is important for the geared rotary actuator (GRA) since its efficiency is strongly dependent on the load case [16].

In order to verify monitoring functions and sensor concepts, the simulation of failure cases is essential. In a transmission system, such as a high-lift actuation system, mechanical disconnection and jamming are, among others, critical failure cases. The disconnection fault model is implemented by adapting the spring-damper component. At a defined failure time both the spring constant and the damping constant are decreased to zero. This value drop is characterized by a time constant as a first-order step response as exemplarily depicted in Figure 3. As a result, no torque is transmitted between the flanges of the component and the separated system parts can be driven independently.

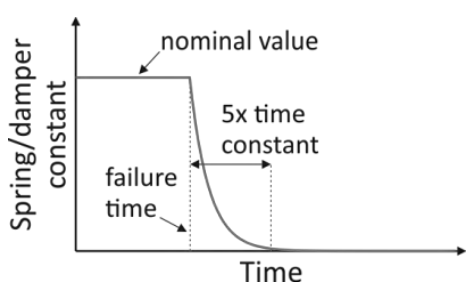

Figure 3: Drop of spring constant and damping constant if disconnection fault is injected

The jamming failure case can be modeled by introducing an additional friction torque. Since the severity of a jamming event may vary, the maximum jamming torque can be defined in the test case specification. Unless this maximum value is exceeded, the jamming event prevents the transmission system from moving. In addition, the jamming fault is characterized by a time constant as illustrated in Figure 4.

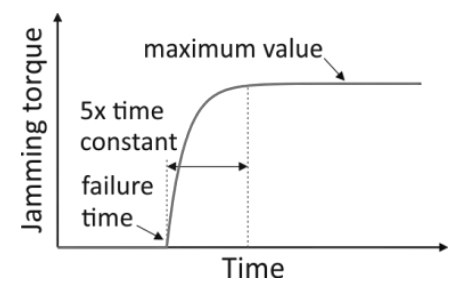

Figure 4: Jamming torque if jamming fault is injected

The interface between the mechanical domain and the electric domain is the electric motor of the ADGB. The transformation between those two domains is defined by the component EMF (electromotive force) as follows:

$$
v=k_{E M F} \cdot \omega
$$

and

$$
\tau=-k_{\tau} \cdot i
$$

where,

$\begin{array}{ll}v: & \text { voltage drop across EMF } \\ i: & \text { armature current } \\ \omega: & \text { angular velocity } \\ \tau: & \text { torque } \\ k_{E M F}: & \text { back EMF constant } \\ k_{\tau}: & \text { torque constant }\end{array}$

If only the back EMF constant is defined, the torque constant is calculated as follows [17]:

$$
k_{\tau}=\frac{3}{2} k_{E M F}
$$

The interface between the hydraulic domain and the mechanical domain is the secondary controlled variable displacement hydraulic motor (VDHM). In this concept the position of the swashplate of the hydraulic motor is 
adjusted to control the motor speed. The implemented component model of the VDHM is based on the linear model presented in [18].

As outlined in the next subsection, equipment models are implemented by connecting corresponding component models.

\subsection{Equipment Models}

The high-lift system of a long-range aircraft modeled in context of this work consists of 14 different equipment types. The corresponding equipment models are depicted in Figure 5 and introduced in more detail below.

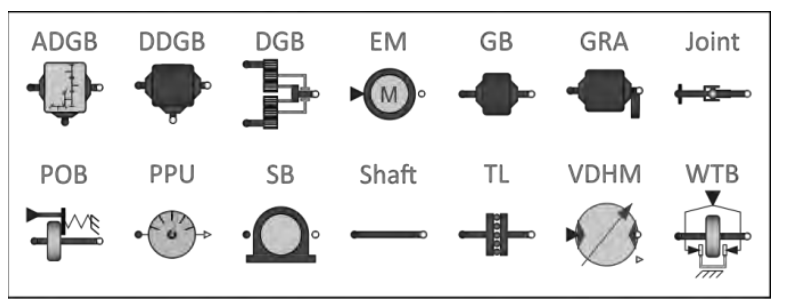

Figure 5: Equipment models of a multi-functional high-lift system

The simplest mechanical equipment model is a shaft consisting of only the components SpringDamper and Inertia as depicted in Figure 6 (left). By extending this model with backlash (ElastoBacklash) and constant efficiency (SlopeEfficiency) the equipment model (transmission) joint results according to the key performance data (Figure 6, right).

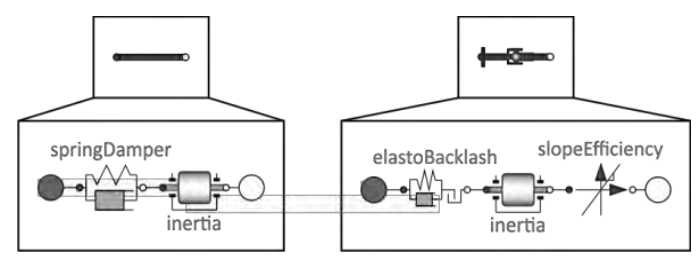

Figure 6: Equipment model of a shaft (left) and a transmission joint (right)

Compared to a joint, a steady bearing (SB) is additionally characterized by speed dependent frictional losses (SlopeEfficiencyDrag) as depicted in Figure 7 (left). The same applies for the equipment model of a gearbox (GB) (Figure 7, right). In addition to the frictional losses (SlopeEfficiencyDrag) the component model GearEfficienyDrag enables the definition of a gear ratio as well as load case dependent efficiency values. For this reason, the model structure of a geared rotary actuator (GRA) is equivalent to the model structure of a GB. In order to model a down drive gearbox (DDGB), the same component models are used. For the DDGB it is assumed that all frictional losses occur in the down drive path.
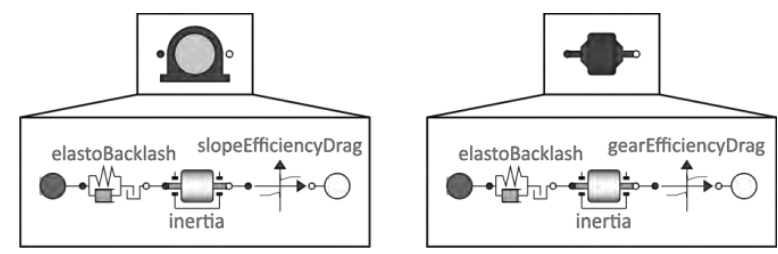

Figure 7: Equipment model of a steady bearing (left) and a gearbox (right)

The fourth type of gearbox is the differential gearbox (DGB) that connects the two hydraulic motors with the transmission system. The present key performance data of the DGB define a moment of inertia at each flange and speed-dependent frictional losses. The general speed summing characteristic of this gearbox with a gear ratio $i_{D G B}$ is specified as follows:

$$
\begin{gathered}
\omega_{\text {out }}=\frac{\omega_{\text {in } 1}+\omega_{\text {in } 2}}{2 \cdot i_{D G B}} \\
\tau_{\text {out }}=2 \cdot i_{D G B} \cdot \tau_{\text {in } 1}=2 \cdot i_{D G B} \cdot \tau_{\text {in } 2}
\end{gathered}
$$

where $\omega$ is the angular velocity and $\tau$ is the torque at input (in) and at output (out) of the gearbox.

In general, brakes of a high-lift transmission system such as wing tip brakes (WTBs) and power-off brakes (POBs) are supplied by at least one hydraulic system. The hydraulic power is used to generate the brake force (WTB) or to release the brake (POB). This behavior is approximated by means of a signal-based brake command with a defined closing time and opening time (BrakeSignal). The brake torque itself is generated by the component Brake from the Modelica Standard Library. The complete model of a WTB is shown in Figure 8.

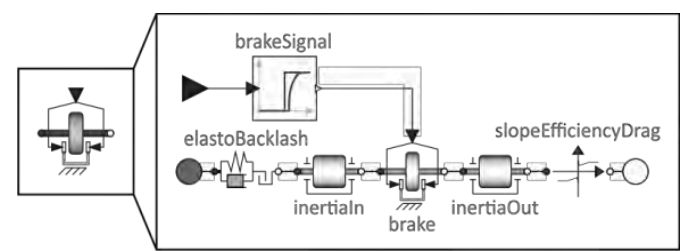

Figure 8: Equipment model of a wing tip brake

In order to mitigate structural damage in case of jamming, each flap drive station is equipped with a torque limiter (TL). In addition, system torque limiters are typically installed on long-range aircraft with high powered drive units. This way, the transmission behind the system torque limiter must be designed to withstand only the threshold torque of the torque limiter and not the maximum torque of the drive unit. The main charac- 
teristics of a torque limiter are represented by a nonlinear torsional stiffness and a brake torque [9]. The torsional stiffness value depends on the applied torque and the relative angular displacement. A classical torque limiter with one lockup stage is described by three discrete modes: nominal operation mode, locking up mode and lockup mode. Each mode is defined by a torque limit and a spring constant value as illustrated in Figure 9. The brake torque is zero in the nominal operation mode. During locking up, the brake torque increases linearly and reaches its maximum value at lockup.

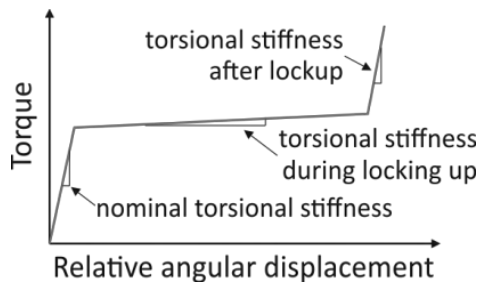

Figure 9: Nonlinear torsional stiffness depending on torque and relative angular displacement

The complete equipment model of a torque limiter is depicted in Figure 10. In addition to the nonlinear spring and brake component, the model is composed of spring-damper and inertia components at input and output as well as of a slope efficiency component.

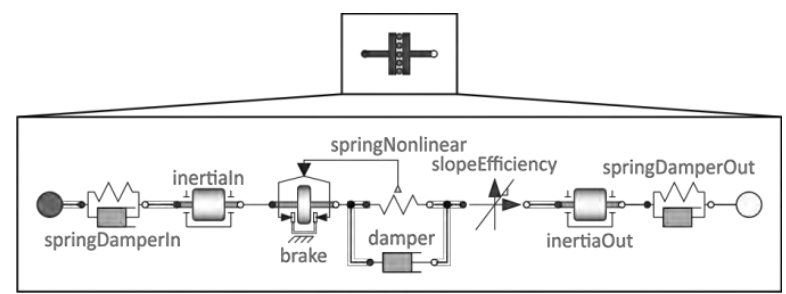

Figure 10: Equipment model of a torque limiter

Depending on the control and monitoring concept of a high-lift system, position sensors at different locations are required. Such position sensors are referred to as position pick-off units (PPUs). The PPU is modeled as an ideal angle sensor with a transmission ratio. The sensor signal is returned in degrees.

As mentioned before, the ADGB enables differential flap setting and represents the main difference compared to conventional high-lift system architectures. This speed summing differential gearbox is driven by the central drive unit when all surfaces should be positioned synchronously (through-drive mode). If only the outer flaps should be moved, the ADGB is driven by an electric motor (EM) and the rest of the transmission system is held by the pressure-off brakes of the central drive unit. For each mode the key performance data specify identical parameter sets similar to the set of the GB (see Figure 7). In contrast to the DGB, the gear ratio of the ADGB depends on which input side is driven. Assuming $i_{T H}$ as defined through-drive gear ratio, the gear ratio $i_{E M}$, when the EM moves the outer flap, is calculated as follows:

$$
i_{E M}=\frac{i_{T H}}{1-i_{T H}}
$$

In order to model the EM in accordance with the key performance data, the above introduced component model EMF is extended by a Resistor, an Inductor and an Inertia from the Modelica Standard Library. This modeling corresponds to a possible simplified representation of a permanent magnet synchronous motor (PMSM) [12]. The resulting equipment model is depicted in Figure 11.

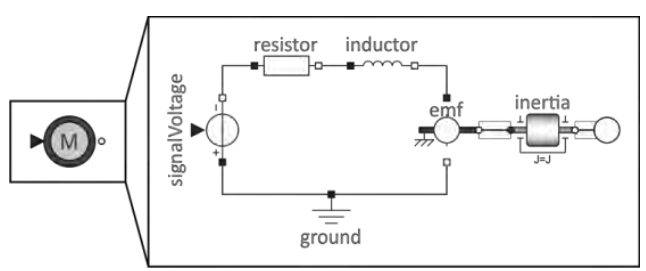

Figure 11: Equipment model of an electric motor (EM)

\section{Virtual Testing of a High-Lift System}

The developed model library enables the evaluation of all nominal operation conditions as well as of characteristic failure cases. By means of validated equipment models and accurate key performance data, such a virtual testing approach could reduce the testing effort of safety-critical flight control systems and provide important results before system level test rigs are available. In order to fully benefit from simulation-based testing, the system modeling effort and the effort of setting up a test case must be as low as possible. For this reason, the library concept is complemented by a preprocessing with a high degree of automatization regarding the test case specific model parameterization. The general concept of the implemented preprocessing is introduced in the next subsection. Afterwards some selected simulation results are presented exemplarily.

\subsection{Preprocessing}

The preprocessing is implemented in the programming language Python. As illustrated in Figure 12, the implemented process can be divided into two main steps: 
1. Creation of a system specific parameter set

2. Creation of a simulation model and executable Modelica scripts

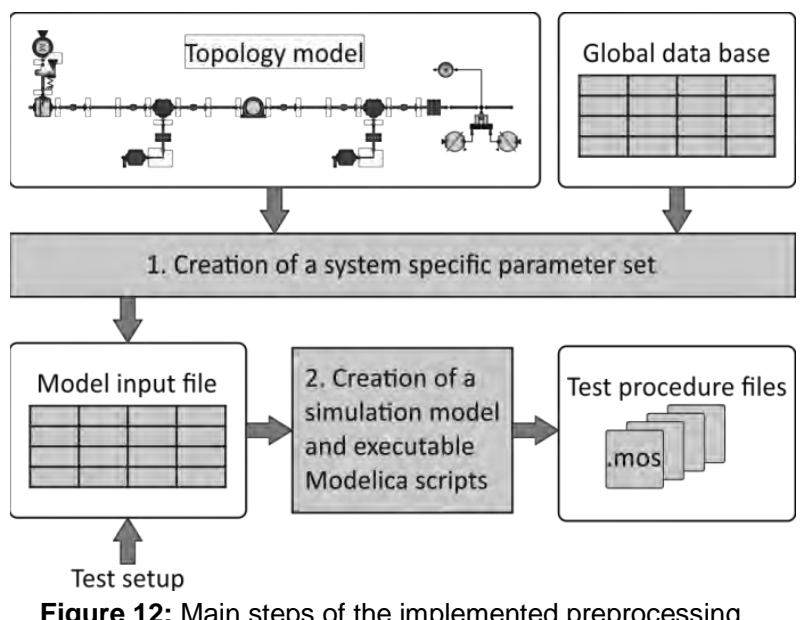

The first step requires the specification of the aircraft and access to the corresponding key performance data. In order to ensure data consistency, it is assumed that the key performance data of different aircraft are stored and managed in one central database. In context of this work, it is termed global data base. By means of a Modelica model the expected system architecture is defined. In addition to the topology information, this model provides an interface between the model library and the key performance data. The equipment class and the equipment name both serve as identifiers for the correct parameter set of the specified aircraft. Finally, all information is stored in a file referred to as model input file.

The generated model input file is the basis for a test campaign. This file is used to adjust the system if necessary (e.g. insertion of faults) and to set up the test procedures. A test procedure is characterized by a simulation time, value type (nominal, minimum, maximum) and environment temperature among others. Depending on the used topology model, it might also be necessary to define the air loads acting on each GRA or to specify the interfaces if the generated model is used for simulation coupling. Afterwards, the model input file contains all information required to generate a simulation model and to parameterize all defined test procedures. The result of the second step is an executable Modelica script for each test case containing the test case specific parameter set and the simulation setup. By executing such a script the defined test case is simulated or a Functional Mock-up Unit (FMU) for co-simulation is generated. All generated and required files are stored in a folder so that the test campaign can be repeated any time, regardless of changes in the model library or the global data base, ensuring fully traceability. In context of virtual testing for certification, the assurance of complete traceability is a crucial aspect that should be covered by suitable simulation data and process management (SDPM) [5,19].

\subsection{Simulation}

The multifunctional high-lift system considered in this work consists of over 100 equipment models. The general structure of such a system model is illustrated in Figure 13. The figure shows the equipment of the left wing between the central drive unit and the ADGB. In the actual simulation model, there are further shafts, joints, steady bearings and gearboxes between the support stations.

In general, the testing of high-lift systems aims at the verification of compliance to the system requirements as well as the correct failure detection and system reaction in such a case. For this purpose, complex system level test rigs are used. Nominal functional tests include, for example, moving the flap to all defined positions at different air loads applied. In order to verify the system behavior in case of a failure, for example, a shaft is replaced by a clutch enabling the simulation of a shaft rupture.

A major advantage of simulation-based testing is the high flexibility. A simulation enables a fast reaction to design changes and the varying of parameter values for a sensitivity analysis or for the evaluation of worst-case scenarios which cannot be normally implemented on a system level test rig. In contrast to a test rig, quantities of interest can be determined at any position enabling, for example, the identification of the most promising sensor position. For this - as discussed earlier - the required data has to be obtained on equipment test benches in the form of key performance data.

The implementation of new monitoring concepts such as an electronic torque limiter requires an accurate knowledge of torque values at all possible operation conditions. As demonstrated in the following, the environment temperature as well as a worst-case scenario might have a major impact on the drive torque and thus on the sensor thresholds. In Figure 14 the torque measured at the output of the central drive unit for different environment temperatures is depicted. The values are normalized to the maximum torque value. Since the 


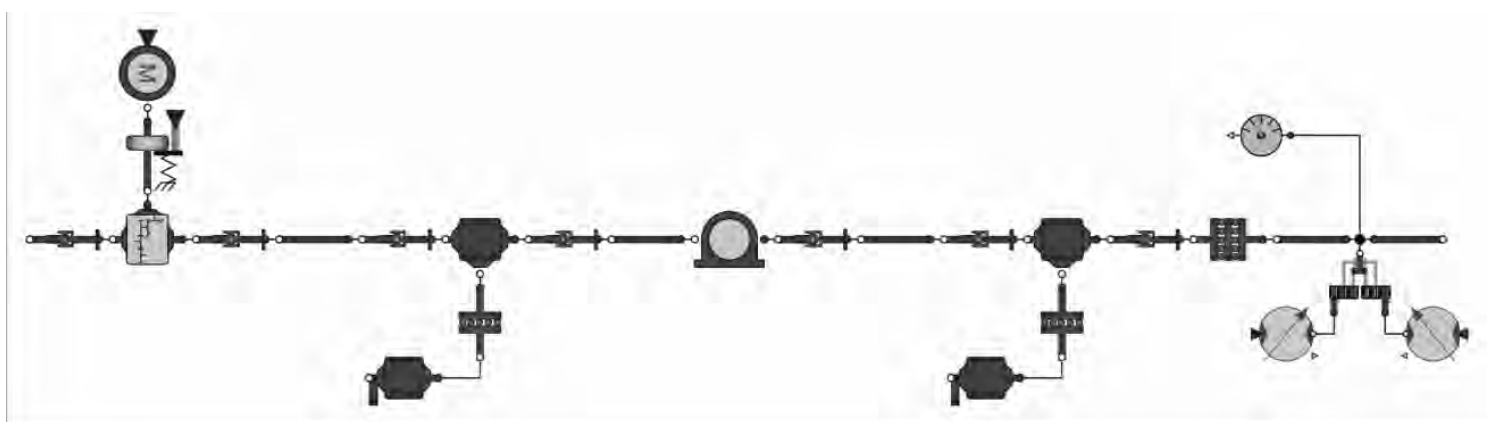

Figure 13: General model structure of a high-lift system

frictional losses increase with decreasing temperature, the necessary drive torque increases with lower temperature. At higher temperatures (here: $+20^{\circ} \mathrm{C}$ ) on the other hand, the low frictional losses lead to a strong oscillation of the drive torque. Moreover, the worst case scenario characterized by maximum frictional losses and minimum efficiency values impacts the resulting drive torque enormously. Such an analysis is essential for the implementation of a robust sensor concept. Nevertheless, not all scenarios can be carried out on a test bench at a reasonable cost or without the risk of damaging the test rig. Assuming that the models and the data are representative, simulation-based testing extends the test scope and enables a comprehensive verification of the system.

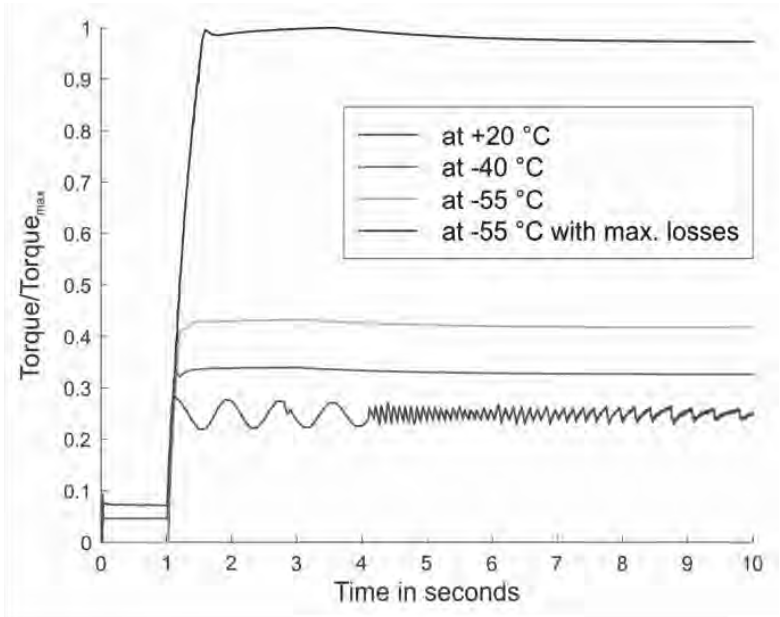

Figure 14: Required drive torque at maximum operation loads depending on environment temperature

Similar effects can be observed by evaluating failure conditions. As mentioned above, disconnection is a typical failure case of a high-lift transmission system. As illustrated in Figure 15, the test setup might have a strong impact on the system behavior and thus the sensor thresholds. The figure shows the measured angle at the central drive unit (FPPU) and at the end of the left wing (APPU). In the first case, the simulation is performed with key performance data for an environment temperature of 20 degrees Celsius. At a simulation time of six seconds a shaft rupture occurs between the inner flap and the outer flap. The applied air loads push the disconnected part in retraction direction. When the defined asymmetry threshold (here: four degrees) is exceeded, the WTB is applied and the drive unit is shut down. In the second case, the same test procedure is conducted with key performance data for an environment temperature of -55 degrees Celsius. In this case the frictional losses are much higher so that the applied air loads are not able to push the disconnected flap surface back. Consequently, the assumed sensor concept fails to detect the failure in this scenario. The importance of such investigations increases when innovative systems are to be developed and implemented.

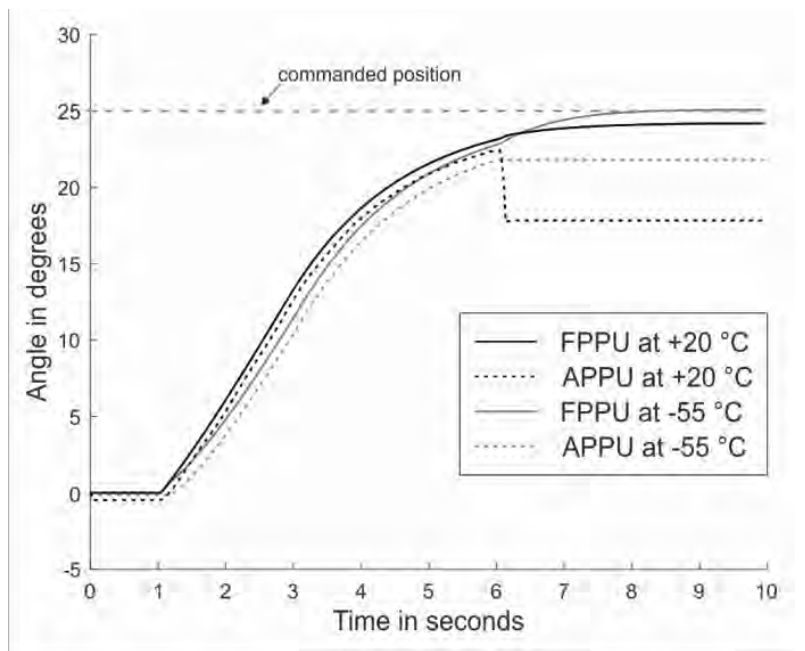

Figure 15: Simulation results of a shaft disconnection at $+20^{\circ} \mathrm{C}$ and $-55^{\circ} \mathrm{C}$

\section{Summary and Outlook}

In the context of this work, a Modelica library enabling the modeling and simulation of multi-functional high- 
lift actuation systems has been developed. Nonlinear models with lumped parameters are implemented based on the so-called key performance data base. In addition to the nominal characteristics, the mechanical faults disconnection and jamming are implemented in the flight control actuation systems library. By determining the key performance data on equipment test benches, simulation-based verification of requirements on overall system level can be performed before system test rigs are available. Besides, the environmental conditions of small equipment test benches can be varied with less effort in contrast to system level test rigs. As illustrated above, the environment temperature might have a strong impact on the system behavior.

In order to generate more representative simulation results, the transmission system will be simulated with the high-lift structure by means of a co-simulation as the next step. Both the flap surfaces and the flap mechanisms are modeled as elements of a multibody system. The co-simulation will be implemented using the Functional Mock-up Interface (FMI).

Acknowledgments. This work was supported by the German Federal Ministry for Economic Affairs and Energy (BMWi) in the framework of the Federal Aeronautical Research Program (LuFo V-3 Phy-ViTeM under the support code 20X1725C).

\section{References}

[1] Moir, I., Seabridge, A. Design and Development of Aircraft Systems. Chichester, UK: John Wiley \& Sons; 2012. 312 p.

[2] Hans, C., Hribernik, K. NFF Special Session - Potentials of Applying Methods, Tools, Processes and Knowledge from Testing in Product Development to the NFF Problem. Procedia CIRP. 2014; 22, 53-58. doi: 10.1016/j.procir.2014.07.131.

[3] Jandaurek, K., Johst, M. Development Trends and Innovations in Aerospace System Testing Using the Example of High-Lift. 55th AIAA Aerospace Sciences Meeting; 2017 Jan; Grapevine, USA. doi: 10.2514/6.2017-0548.

[4] Langermann, R. Beitrag zur durchgängigen Simulationsunterstützung im Entwicklungsprozess von Flugzeugsystemen [dissertation]. Technische Universität Braunschweig; 2009.

[5] Ulmer, T., Amin, J. Virtual Testing of High Lift Systems. SAE Technical Paper. 2013; 2013-01-2280. doi: 10.4271/2013-01-2280.

[6] Valdivia-Guerrero, V., Foley, R., Riverso, S., et al. Modelling and Simulation Tools for Systems Integration on Aircraft. SAE Technical Paper. 2016; 2016-01-2052. doi: 10.4271/2016-01-2052.

[7] Schäfer, A., Hollmann, R., Bertram, O. Process for Virtual Design and Testing of Flight Control Actuation Systems. 68. Deutscher Luft- und Raumfahrtkongress; 2019 Oct; Darmstadt, Germany.

[8] Fritzson, P. Principles of object-oriented modeling and simulation with Modelica 3.3: A cyber-physical approach. Piscataway, US: Wiley-IEEE Press; 2014, 1256 p.

[9] Pfennig, M., Thielecke, F. Implementation of a Modelica Library for Simulation of High-Lift Drive Systems. 6th International Modelica Conference; 2008 March; Bielefeld, Germany.

[10] Lulla, C. Functional Flexibility of the A350XWB High Lift System. 60. Deutscher Luft- und Raumfahrtkongress; 2011 Sept; Bremen, Germany.

[11] Strüber, H. The Aerodynamic Design of the A350 XWB900 High Lift System. 29th International Congress of the Aeronautical Sciences; 2014 Sept; St. Petersburg, Russia.

[12] Schäfer, A., Schmid, M. Analysis of the Effects of Modeling Depth and Parameter Uncertainties on the System Behavior of a Multifunctional High Lift Actuation System. SAE Technical Paper. 2018; 2018-01-1918. doi: 10.4271/2018-01-1918.

[13] Tiller, M. Introduction to Physical Modeling with Modelica. Boston, US: Springer; 2001, 345 p.

[14] Modelica Standard Library. https://github.com/modelica/ModelicaStandardLibrary, viewed 24 August 2020.

[15] Olsson, H., Åström, K. J., De Wit, C. C., Gäfvert, M., Lischinsky, P. Friction Models and Friction Compensation. European Journal of Control. 1998; 4(3), 176-195. doi: 10.1016/S0947-3580(98)70113-X.

[16] Wang, A., Gitnes, S., El-Bayoumy, L. The Instantaneous Efficiency of Epicyclic Gears in Flight Control Systems. Journal of Mechanical Design. 2011; 113(5). doi: 10.1115/1.4004001.

[17] Olaf Cochoy. Investigations for the Synchronized Operation of a Hybrid Actuator Configuration in Redundant Flight Control Systems [dissertation]. Hamburg University of Technology; 2009.

[18] Geerling, G. Entwicklung und Untersuchung neuer Konzepte elektrohydraulischer Antriebe von FlugzeugLandeklappensystemen [dissertation]. Hamburg University of Technology; 2003.

[19] Ulmer, T., Amin, J. Virtual Testing of High Lift Systems. NAFEMS World Congress 2013; 2013 June; Salzburg, Austria.

\section{Definitions/Abbreviations}

ADGB - Active differential gear box

DDGB - Down drive gearbox 
DGB - Differential gearbox

EM - Electric motor

EMF - Electromotive force

FMI - Functional Mock-up Interface

FMU - Functional Mock-up Unit

GB - Gearbox

GRA - Geared rotary actuator

OEM - Original equipment manufacturer

PMSM - Permanent magnet synchronous motor

POB - Power-off brake

PPU - Position pick-off unit

SDPM - Simulation data and process management

SB - Steady bearing

$\mathrm{TL}$ - Torque limiter

VDHM - Variable displacement hydraulic motor

WTB - Wing tip brake 